\title{
MODELO MATEMÁTICO DE RODA DE REAÇÃO À BAIXA VELOCIDADE, ESTIMAÇÃO DE PARÂMETROS E CONTROLE DE ATITUDE DE MESA DE MANCAL A AR COM RODA.
}

\author{
GUILHERME A. SIQUELI ${ }^{1}$, VALDEMIR CARRARA ${ }^{1}$, WILIAN L. CAMPESATO ${ }^{2}$. \\ 1. Divisão de Mecânica Espacial e Controle, Instituto Nacional de Pesquisas Espaciais \\ E-mails: guilherme.siquelieinpe.br, val@dem.inpe.br
}

2. Centro de Engenharia e Ciências Sociais, Universidade Federal do ABC

E-mails: wilian.campesato@ufabc.edu.br

\begin{abstract}
In this work is performed a characterization, estimation of parameters and of the mathematical model of a reaction wheel of a table bearing the air. The nonlinear dynamics of the wheel at low speeds subject are analyzed and compensation methods angular velocity and current are proposed in order to linearize the model of the wheel. A classic PID control is proposed and designed either manually or through Ziegel-Nichols tuning and their performances are compared.
\end{abstract}

Keywords— Reaction wheel, control, parameters estimation, simulation.

Resumo - Neste trabalho é realizado uma caracterização, estimação de parâmetros e do modelo matemático de uma roda de reação de uma mesa de mancal a ar. A dinâmica não linear da roda sujeita a baixas velocidades, é analisada e métodos de compensação de corrente e velocidade angular são propostos a fim de linearizar o modelo da roda. Um controle clássico PID é proposto e projetado tanto manualmente como através de sintonização por Ziegler-Nichols e suas performances são comparadas.

Palavras-chave— Roda de reação, controle, estimação de parâmetros, simulação.

\section{Introdução}

O controle de atitude de satélites artificiais é um vasto e importante campo de pesquisa. Dentre os diversos mecanismos e atuadores que podem ser embarcados em um satélite artificial, as rodas de reação apresentam grande destaque, pelo fato de apresentarem uma dinâmica satisfatória, mesmo sujeita a ação de pequenas perturbações. Rodas de reação são amplamente utilizadas em satélites que apresentam controle em três eixos (NUDEHI et al., 2008).

Rodas de reação são motores DC sem escovas e com grande inércia. Tais dispositivos foram fundamentais para o desenvolvimento da engenharia aeroespacial e da exploração espacial e estudos precursores, como o de Froelich e Patapoff (1959), foram fundamentais para o entendimento da dinâmica de tais dispositivos.

O Brasil dispõe de rodas de reação em laboratório, como a alocada no laboratório de simulação (LabSim) do Instituto Nacional de Pesquisas Espaciais (INPE). O comportamento desta estrutura foi estudado por Carrara e Milani (2007) que propuseram um modelo matemático que descreve a dinâmica da roda através de uma equação diferencial linear, na qual a mesma é sujeita a torques provenientes de atritos internos de dissipação tanto viscosa, como de Coulumb (atrito de partida) e torques externos aplicados sobre a roda. Carrara e Milani (2007) projetaram ainda controles PID e avaliaram sua performance no controle de uma mesa de mancal a ar, na qual a roda mencionada anteriormente se encontrada instalada.

Carrara (2010) realizou uma comparação experimental entre formas de controle de atitude com o uso de rodas de reação e estudou o comportamento de uma roda quando a mesma opera em velocidades muito baixas, decaindo ou subindo sua velocidade e propositalmente invertendo seu sinal.

Tendo em vista a necessidade eventual de operação da roda de reação em baixas velocidades, sua dinâmica não linear e a incompatibilidade com os modelos apresentados na literatura, este trabalho apresenta um modelo para a roda de reação em baixas velocidades e o controle de uma mesa de mancal a ar experimentalmente com base no modelo discutido.

\section{Materiais e Métodos}

\subsection{Materiais}

Os equipamentos que compõem a mesa de mancal de ar do Laboratório de Simulação (LabSim) do INPE e que foram utilizados para a análise experimental desde trabalho, são listados a seguir:

- Mesa de mancal aerostático $(650$ mm de diâmetro)

- Roda de Reação da "SunSpace ${ }^{\circledR}$ (The SunSpace, Inc., Techno Park, Stellenbosch, South Africa)" 
- Giroscópio tipo FOG da SunSpace

- Radio-modem da "FreeWave ${ }^{\circledR}$ (FreeWave Technologies, Inc., Boulder, CO)”

- Bateria de $12 \mathrm{~V}$

- Computador para telemetria da "National Instruments @ (National Instruments, Inc., Austin, Texas, U.S.A)"

O aparato experimental é apresentado na Fig. 1. Observa-se que a fim de minimizar a presença de torques externos, a comunicação realizada entre a mesa de mancal e o computador que gera os telecomandos é realizada via radio-modem e não por fios elétricos.

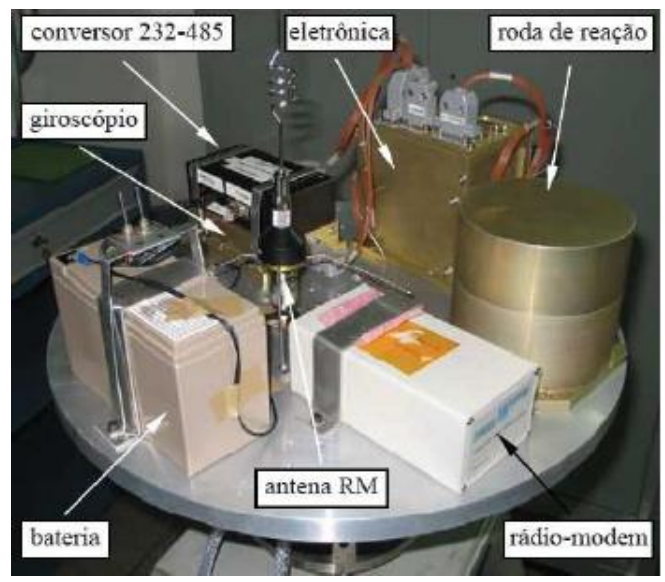

Figura 1. Equipamento montado

\subsection{Métodos}

Os parâmetros mais relevantes da roda de reação, que podem ser estimados experimentalmente, são os coeficientes de atrito (viscoso e de Coulomb).

O coeficiente de atrito viscoso é obtido através da análise do decaimento da velocidade angular da roda, caso esta não esteja sujeita a torques externos.

O coeficiente de atrito de Coulomb é obtido através do incremento de corrente de armadura sobre o motor e observação do instante em que a roda começa a apresentar algum movimento. Os dados são obtidos pelos sensores instalados na estrutura.

$\mathrm{O}$ parâmetro associado à energia potencial acumulada pela roda antes da mesma partir é calculado considerando duas regiões de operação da roda com modelos diferentes com continuidade de energia na fronteira (ponto de transição entre os modelos).

\section{Modelagem da Roda de Reação}

O modelo matemático, que descreve o comportamento dinâmico da roda de reação, considera a presença de torques provenientes dos atritos viscoso e de Coulomb além do torque elétrico exercido sobre a roda que faz com que a mesma altere sua velocidade angular. O modelo apresentado por Carrara (2010) é dado pela Eq. (1). Uma análise mais detalhada sobre os atritos dinâmicos presentes na roda pode ser encontrado em Carrara, Silva e Kuga (2012).

$$
k_{m} i=J \dot{\omega}+b \omega+c \operatorname{sgn}(\omega)
$$

A solução desta equação é dada pela Eq. (2).

$$
\omega(t)=\alpha e^{-\beta t}-\gamma
$$
Eq. (3)

Em que os parâmetros $\alpha, \beta$ e $\gamma$ são dados pela

$$
\begin{gathered}
\alpha=\omega_{0}+\gamma \\
\beta=b / J \\
\gamma=c / b=\omega_{0} \frac{e^{\frac{-b t}{J}}-e^{\frac{-b t_{f}}{J}}}{1-e^{\frac{-b t_{f}}{J}}}
\end{gathered}
$$

Com as devidas substituições a Eq. (2) se torna:

$$
\omega(t)=\omega_{0} \frac{e^{\frac{-b t}{J}}-e^{\frac{-b t_{f}}{J}}}{1-e^{\frac{-b t_{f}}{J}}}
$$

Através da solução analítica do modelo (Eq. (4)) é possível estimar o parâmetro b/J, ajustando-o numericamente, através do "Software MATLAB ${ }^{\circledR}$ (The MathWorks, Inc., Natick, Massachusetts, U.S.A)", de forma que esta solução (com velocidade angular inicial $\omega_{0}=403,6 \mathrm{rad} / \mathrm{s}$ e tempo total até atingir velocidade nula de 366,92 s) seja o mais próximo possível da curva de decaimento obtida experimentalmente. Após os devidos ajustes encontra-se um valor de 0,004 para o parâmetro b/J. As curvas experimental e analítica, após ajuste do parâmetro, são ilustradas na Fig. 2.

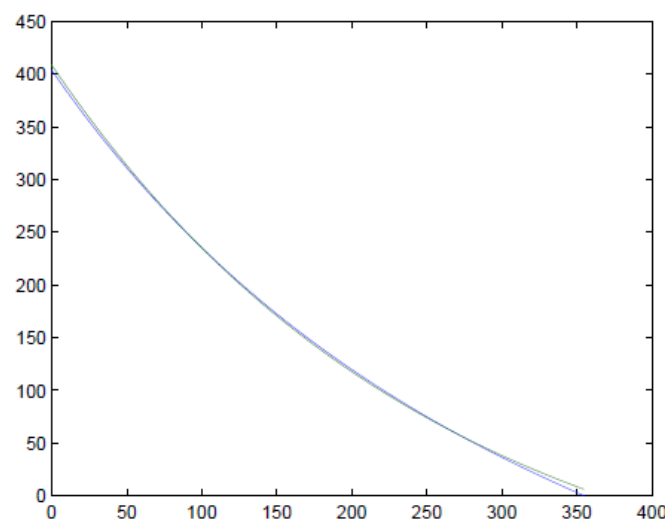

Figura 2. Estimativa do parâmetro b/J 
Adotando-se a inércia da roda como sendo conhecida (fornecida pelo fabricante) de valor $1,510^{-3}$ kg. $\mathrm{m}^{2}$, tem-se que $\mathrm{b}=5,99410^{-6} \mathrm{Nm} . \mathrm{s}$.

$\mathrm{O}$ atrito de Coulumb, obtido através de iteração paulatina da corrente de armadura e observação do comportamento da velocidade da roda, apresenta valor de $0,00104 \mathrm{Nm}$.

A constante de proporcionalidade do motor $\left(\mathrm{k}_{\mathrm{m}}\right)$ é obtida através da resposta em regime permanente do motor. Através de uma curva corrente por velocidade (Fig.3), obtida através de uma iteração de corrente sobre o motor e observação do valor de velocidade angular em regime permanente para cada valor de corrente, é possível estimar o valor $\mathrm{k}_{\mathrm{m}}$ pela inclinação da curva, que se aproxima de uma reta obtida através do método de mínimos quadrados. $\mathrm{O}$ valor obtido para $\mathrm{k}_{\mathrm{m}}$ foi de $0,0311 \mathrm{Nm} / \mathrm{A}$.

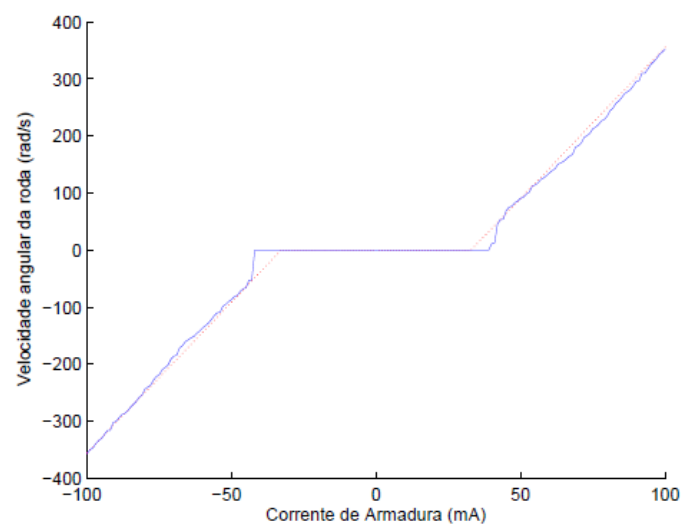

Figura 3. Curva experimental da roda de reação (traço azul) e curva dada pelo modelo matemático (traço vermelho)

O modelo matemático completo da roda é dado então pela Eq. (5).

$$
\begin{gathered}
k_{m} i=J \dot{\omega}+b \omega+c \operatorname{sgn}(\omega) \quad p / \omega>0 \quad e \quad p / i<\frac{c}{k_{m}} \\
k_{m} i=J \dot{\omega}+b \omega+c \operatorname{sgn}(\omega) \quad p / \omega<0 \quad e \quad p / i>\frac{-c}{k_{m}} \\
\omega=0 \quad p / \frac{-c}{k_{m}}<i<\frac{c}{k_{m}}
\end{gathered}
$$

Observa-se que para valores de corrente entre -40 e $40 \mathrm{~mA}$ (aproximadamente) a roda não apresenta movimento. A dinâmica da roda logo após a partida, em baixas velocidades apresenta uma velocidade resposta diferente da região linear de altas velocidades; Por este motivo é muitas vezes difícil identificar exatamente o ponto de partida da roda e predizer sua dinâmica nesta região de operação em baixa velocidade.

De fato, com o aumento da corrente de armadura, partindo de zero, observa-se que a roda parte (em ambos os sentidos) com correntes mais elevadas (em módulo) do que o esperado pelo modelo linear. Este efeito pode ser modelado através de uma componente de torque resistivo em baixas velocidades que armazena energia potencial da roda até um valor limite.

O modelo para a dinâmica em baixas velocidades é dado pela Eq. (6).

$$
k_{m} i=J \dot{\omega}+b \omega+G \int \omega d t+c \operatorname{sgn}(\omega)
$$

A terceira parcela do lado direito da Eq. (6) é justamente a que contribui para o armazenamento de energia da roda antes de sua partida. A função de transferência da equação diferencial acima é dada por:

$$
\Omega(s)=\frac{k_{m} i-c}{J s^{2}+b s+G}
$$

Utilizando o método de Heaviside e em seguida a transformada inversa de Laplace a solução da Eq. (6) é dada pela Eq. (8).

$$
\omega(t)=\frac{J\left(k_{m} i-c\right)}{\sqrt{\left(b^{2}-4 J G\right)}} e^{\frac{-b t}{2 J}}\left[e^{\frac{\sqrt{\left(b^{2}-4 J G\right)}}{2 J} t}-e^{\frac{-\sqrt{\left(b^{2}-4 J G\right)}}{2 J} t}\right\rfloor
$$

Dois resultados de especial interesse são aqueles em que a expressão (Eq. (8)) é avaliada em $\mathrm{t}=0 \mathrm{e}$ $\mathrm{t} \rightarrow \infty$, ambas apresentando valor nulo. Este resultado é de se esperar uma vez que a roda de fato não apresenta movimento, com este modelo, até atingir um valor de corrente tal que vença a energia máxima que pode ser acumulada por ela. Após a partida da roda, observa-se que a inclinação média da curva é superior ao valor encontrado anteriormente. Em outras palavras, o parâmetro $\mathrm{k}_{\mathrm{m}} / \mathrm{b}$ apresenta valor elevado.

$\mathrm{O}$ parâmetro $\mathrm{G}$ é obtido através da análise da continuidade da energia total do sistema. Esta quantidade para alta velocidade ( $\left.E_{1}\right)$ é dada pela Eq. (9). Já para o modelo considerando operação da roda em baixa velocidade é dada pela Eq. (10).

$$
\begin{aligned}
& E_{1}=\frac{1}{2} J[\omega(t)]^{2} \\
& E_{1}=\frac{1}{2} J\left[\frac{k_{m} i-c}{b}+\left[\omega_{0}-\frac{k_{m} i-c}{b}\right] e^{\left.\frac{-b\left(t_{f}-t_{0}\right)}{J}\right]^{2}}\right.
\end{aligned}
$$




$$
\begin{aligned}
& E_{2}=\frac{1}{2} J[\omega(t)]^{2}+\frac{1}{2} G\left[\int \omega(t) d t\right]^{2} \\
& E_{2}=\frac{1}{2} J\left[\frac{J\left(k_{m} i-c\right)}{\sqrt{\left(b^{2}-4 J G\right)}} e^{\frac{-b t}{2 J}}\left[e^{\frac{\sqrt{\left(b^{2}-4 J G\right) t}}{2 J}}-e^{\frac{-\sqrt{\left(b^{2}-4 J G\right) t}}{2 J}}\right]\right]^{2}+ \\
& \frac{1}{2} G\left[\int\left[\frac{J\left(k_{m} i-c\right)}{\sqrt{\left(b^{2}-4 J G\right)}} e^{\frac{-b t}{2 J}}\left[e^{\frac{\sqrt{\left(b^{2}-4 J G\right) t}}{2 J}}-e^{\frac{-\sqrt{\left(b^{2}-4 J G\right) t}}{2 J}}\right]\right] d t\right]^{2}
\end{aligned}
$$

Para satisfazer a continuidade da função energia total do sistema na transição entre os modelos o valor de $\mathrm{G}$ encontrado é de $6,45510^{-14} \mathrm{Nm}$. O ponto de transição encontrado apresenta corrente de armadura de $42 \mathrm{~mA}$ e $\mathrm{E}=1,4742 \mathrm{~J}$.

Neste caso a roda de reação apresenta regiões diferenciadas de operação, regidas por modelos matemáticos diferentes. Para baixas velocidades o modelo que descreve seu comportamento é dado pela Eq. (6) e para altas velocidades o termo integral da velocidade angular não é considerando sendo assim válido o modelo dado pela Eq. (1).

\section{Projeto do controle PID e métodos de compensação}

A seguir é projetado um controle PID para controle de atitude da mesa de mancal a ar, que é composto por três parcelas, sendo uma proporcional ao erro (quantidade entre o valor de referência adotado e o valor atual da posição angular), outra proporcional a variação temporal do erro e uma última proporcional a acumulação (integral) temporal do erro. A ação de controle é dada por:

$$
u(t)=k_{p}\left\lfloor\int \omega(t) d t+\frac{1}{T_{i}} \iint \omega(t) d t+T_{d} \omega(t)\right\rfloor
$$

em que $T_{i}$ é o tempo integral e $T_{d}$ é o tempo derivativo.

Ajustando manualmente os parâmetros do controlador $\left(\mathrm{k}_{\mathrm{p}}, \mathrm{T}_{\mathrm{d}}, \mathrm{T}_{\mathrm{i}}\right)$ de forma que o sistema apresente um máximo sobressinal pouco elevado e um tempo de acomodação satisfatório tem-se $\mathrm{k}_{\mathrm{p}}=0,04, \mathrm{~T}_{\mathrm{d}}=5 \mathrm{e} \mathrm{T}_{\mathrm{i}}$ $=40$.

Sintonizando o controlador através do método de Ziegler-Nichols, obtém-se o valor de ganho crítico como sendo $\mathrm{k}_{\mathrm{cr}}=0,2$ e o período da resposta puramente oscilatória de $\mathrm{P}_{\mathrm{cr}}=23 \mathrm{~s}$ finalmente os ganhos são dados por $\mathrm{k}_{\mathrm{p}}=0,12, \mathrm{~T}_{\mathrm{d}}=2,875 \mathrm{e} \mathrm{T}_{\mathrm{i}}=11,5$. As respostas da mesa de mancal a ar, sujeito a controles PID ajustados pelos dois métodos anteriores, com condição inicial de $-90^{\circ}$ e referência em $0^{\circ}$, são ilustrados na Fig. 4.

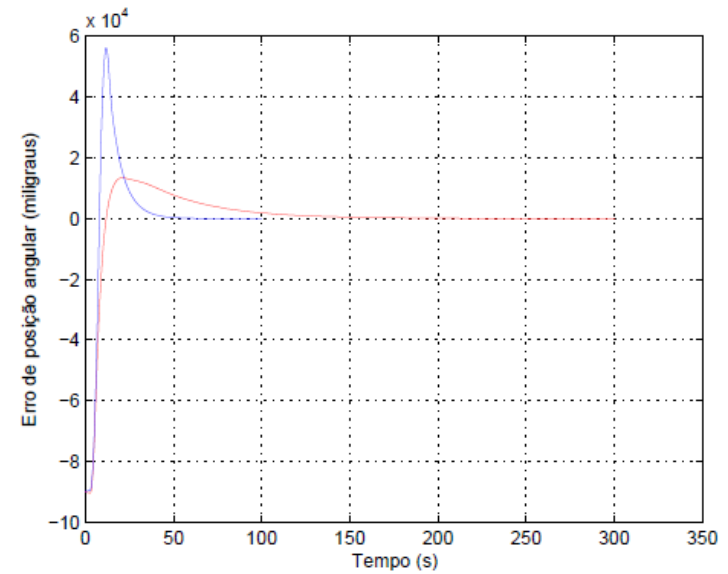

Figura 4. Variação temporal do erro de posição angular da mesa de mancal a ar com controle PID ajustado manualmente (traço em vermelho) e sintonizado por Zieghel-Nichols (traço em azul).

É possível observar que o controle sintonizado apresenta um tempo de acomodação menor à custa de um sobressinal consideravelmente maior.

Utilizando os parâmetros do controle ajustado manualmente e inserindo um torque externo constante através de um cooler instalado sobre a plataforma de forma que a velocidade angular da roda caia linearmente, é possível avaliar o comportamento da corrente na roda e a evolução do erro da mesa de mancal a ar. Na Fig. 5 são ilustradas as variações temporais da velocidade angular e corrente da roda e o erro de atitude da mesa de mancal.

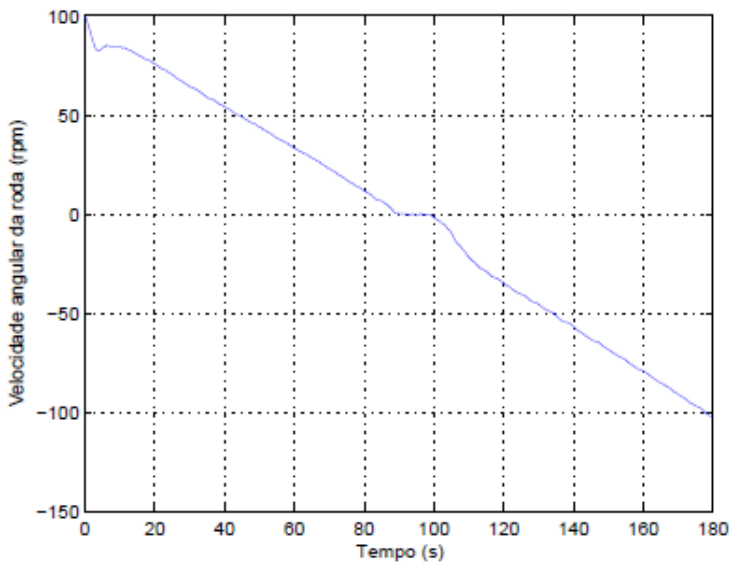

(a) 


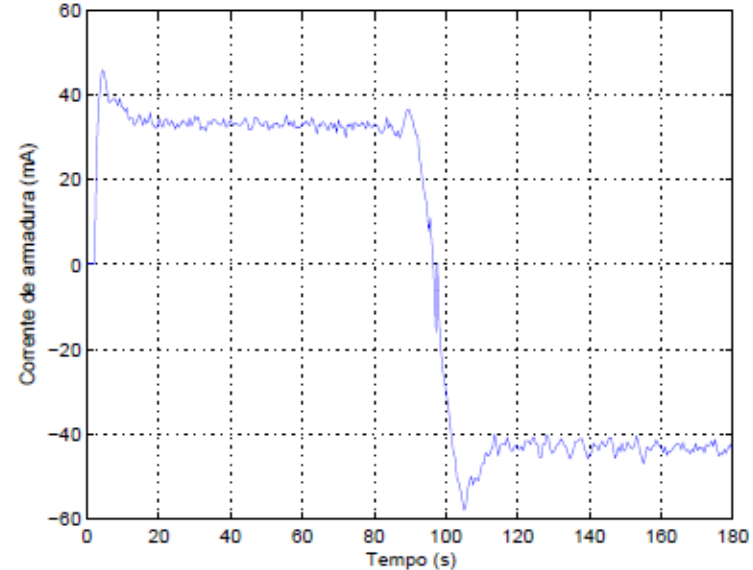

(b)

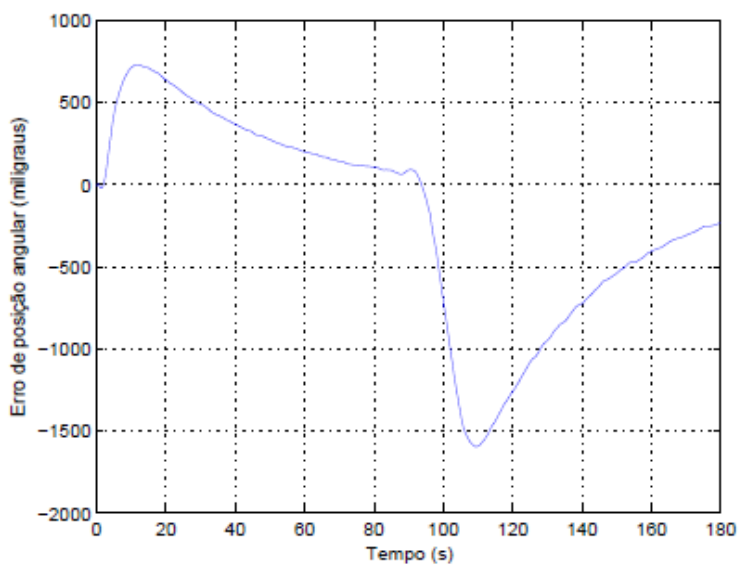

(c)

Figura 5. Variação temporal da posição angular (a), da corrente de armadura na roda (b) e do erro de posição angular da mesa de mancal a ar (c) para baixa velocidade, sem compensação.

Observa-se uma elevação expressiva do erro de posição angular quando a roda cruza $0 \mathrm{rpm}$ e o chaveamento da corrente de estabilização de baixa velocidade de um valor positivo para um valor negativo.

Se o controle incluir as compensações de torque devido à zona morta e a dinâmica diferenciada da região de baixa velocidade, o erro de atitude da mesa de mancal reduz significativamente (de aproximadamente $1,5^{\circ}$ para $0,4^{\circ}$ ). As evoluções temporais da velocidade angular e corrente da roda são ilustradas na Fig. 6 assim como o erro de atitude da mesa de mancal.

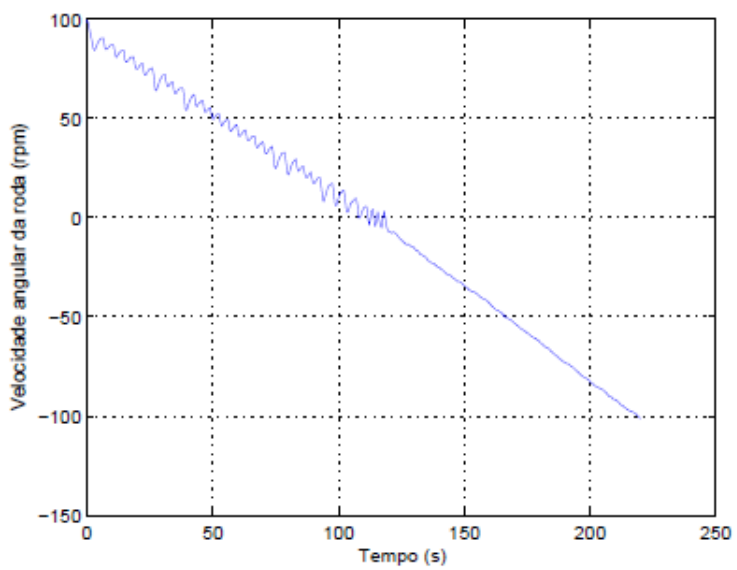

(a)

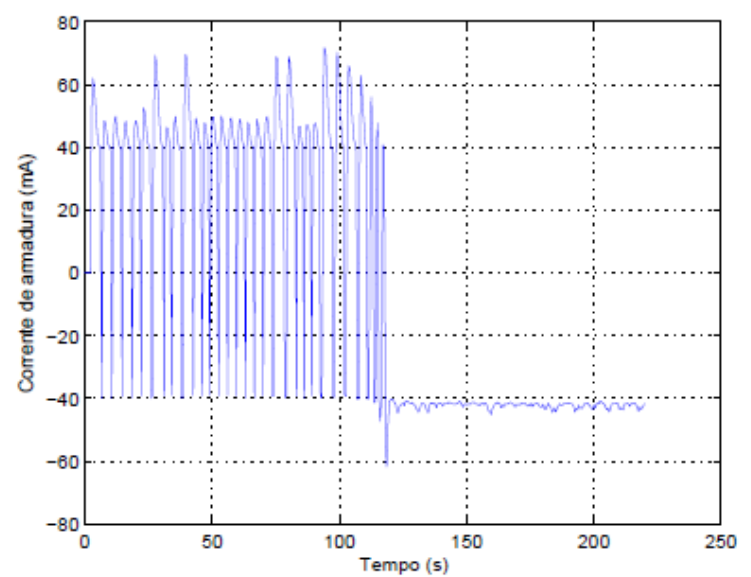

(b)

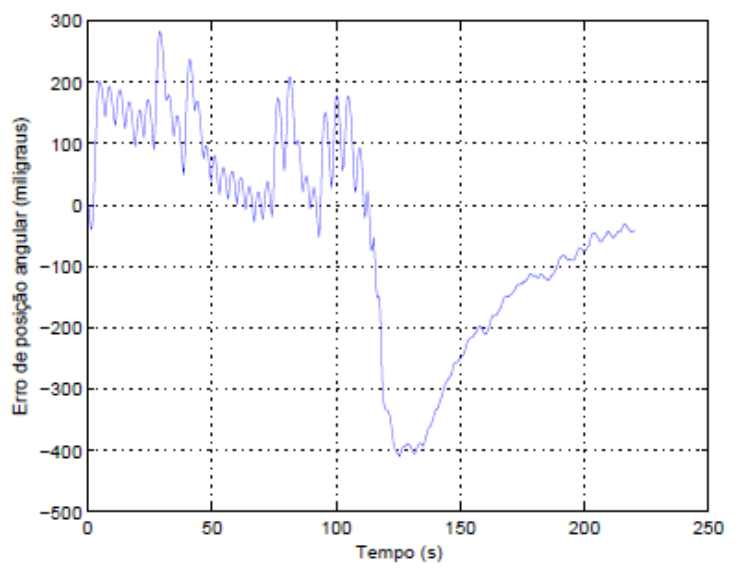

(c)

Figura 6. Variação temporal da posição angular (a), da corrente de armadura na roda (b) e do erro de posição angular da mesa de mancal a ar (c) para baixa velocidade, com compensação.

\section{Conclusão}

A dinâmica de uma roda de reação é predominantemente não linear, especialmente para operação em baixas velocidades. $\mathrm{O}$ modelo que descreve o comportamento da roda baseado apenas nas contribuições de torques externos aplicados e torques de atrito viscoso e de Coulumb é insuficiente para des- 
crever a dinâmica da roda em baixas velocidades, uma vez que a dinâmica nesta região é diferenciada.

A estimação de parâmetros do modelo referente aos atritos presentes pode ser realizada através dos dados experimentais da roda. Já o parâmetro relacionado com a energia acumulada pela roda em baixas velocidades antes de partir pode ser estimado considerando conservação de energia no ponto de transição entre os modelos.

O controle PID apresentou resposta satisfatória no controle de atitude da mesa de mancal a ar, porém apresentou erro significativo quando a roda inverteu o sinal de sua velocidade. Neste caso, a compensação dinâmica na lei de controle dos efeitos da zona morta e da dinâmica em baixa velocidade reduziu o erro máximo, na inversão, na ordem de $75 \%$.

\section{Agradecimentos}

Os autores agradecem ao CNPq e ao INPE através do Programa PIBIC, pela oportunidade de ter realizado este projeto nas dependências do Instituto e seu laboratório LabSim nos anos 2009-2010.

\section{Referências Bibliográficas}

Carrara, V. (2010). Experimental comparasion between reaction wheel atitude controller strategies. Journal of Aerospace Engineering, Sciences and Applications., vol. II, $\mathrm{n}^{\circ}$ 2. DOI: 10.7446/jaesa.0202.01

Carrara, V. and Milani, P. G. (2007). Controle de uma Mesa de Mancal a Ar de um Eixo Equipada com Giroscópio e Roda de Reação.V Simpósio Brasileiro de Engenharia Inercial.

Carrara, V. and Silva, A. G. and Kuga, H. K. (2012). A dynamics friction model for reaction wheels. IAA Conference on Dynamics and Control of Space Systems.

Froelich, R. W. and Patapoff, H. (1959). Reaction Wheel Attitude Control for Space Vehicles.

Nudehi, S. S. and Farooq, U. and Alasty, A. A and Issa, J. (2008). Satellite attitude control using three reaction wheels. 2008 American Control Conference. DOI: 10.1109/ACC.2008.4587262 